\title{
ATUAÇÃo dA ENFERMAGEM EM UM SISTEMA DE SAÚDE COMUNITÁRIA *
}

\author{
** Maria Conceição Resende \\ ** Aurea Moretti Pires \\ ** Maria José Galti
}

\begin{tabular}{l|l|} 
& RBEn/02 \\
\hline
\end{tabular}

RESENDE, M. C. e Colaboradoras - Atuação da enfermagem em um sistema de saúde comunitária. Rev. Bras. Enf.; DF, 33 : 132-156, 1980.

\section{I - INTRODUÇÃO}

Com base na concepção científica que rege os princípios de assistência à comunidade, para a qual se voltam, na atualidade os governos e a equipe de saúde, inclusive com recomendação da Organização Mundial de Saúde, valorizando a prevenção de doenças através da promoção da saúde, assim também com base na orientação cristã de promoção global do indivíduo integrado em sua comuñidade, orientação essa que norteia a Pontifícia Universidade Católica de Campinas (PUCC) e, ainda, visando a formação de novos campos de estágio de Saúde Pública, as Faculdades de Ciências da Saúde da PUCC uniram-se para programar e proporcionar assistência à população dos bairros próximos ao Hospital "Dr. Celso
Pierro", da PUCC, ora em fase de montagem, a saber: Jardim Garcia, Jardim Londres e Vila Castelo Branco.

Inicialmente foi realizada uma pesquisa na área visando o conhecimento de características da Ecpulação e com base nos resultados, foi elaborado um programa de integração multiprofissional e docente assistencial para a comunidade.

Partindo da assistência oferecida no Ambulatório I - "Dr. Joaquim de Paula Barreto Fonseca", montado no Centro dos bairros a serem atendidos, foram desenvolvidos programas de Puericultura, Pré-Natal, Saúde Escolar e Controle de Hipertensos.

Para futuro próximo está programado o agrupamento de alcóolatras, diabéticos e toxicomanos, bem como 0 . controle de pacientes portadores de

- Trabalho apresentado no XXXI CBEn, em Fortaleza-CE, 1979, e ocupou o terceiro lugar entre os melhores temas-livre.

* Professoras de Enfermagem da Pontifícia Universidade de Campinas - PUC - Campinas-SP. 
RESENDE, M.C. e Colaboradoras - Atuaçãj da enfermagem em um sistema de saúde comunitária. Rev. Bras. Enf.; DF, $33: 132-156,1580$.

Hanseníase que estão sendo detectados na comunidade.

\section{II - HISTÓRICO}

A penetração na comunidade efetuada por docentes e alunos das Faculdades de Ciências Médicas, Enfermagem e Serviço Social, iniciou-se com a pesquisa realizada em novembro de 1977 , sendo que a população mostrou-se arredia e desconfiada e os alunos, procedentes de uma classe socialmente elitizada, demonstravam insegurança e receio nesse contato inicial com uma classe social carente de recursos materiaiis e sócio-culturais.

Em fevereiro de 1978 os profissionais da Pontifícia Universidade Católica de Campinas, iniciaram atuação junto à comunidade em conjunto com - Departamento de Serviço Social da Prefeitura Municipal de Campinas que atuava anteriormente, através de Sociedades de Amigos de Bairro e de grupos comunitários. Assim a Faculdade de Enfermagem, contando com enfermeiras do Departamento de Saúde Pública, alunos de Graduação em Enfermagem e uma Assistente Social, iniciou Visitação Domiciliária para puérperas e gestantes, atuou em um grupo de aposentados, oferecendo: Orientação econômico-social, controle e orientação de saúde, encaminhamento para consulta médica, e realização de pós-consulta. Houve tentativa de formar grupos de gestantes e donas-de-casa que não tiveram continuidade nas reuniōes, provavelmente porque não era of erecida uma assistência médica contínua e o interesse manifestado pelos membros da comunidade girava em torno da necessidade de montar um Ambulatório na comunidade, já que a mesma não contava com recursos locais de saúde e também porque, sendo área periférica, localiza-se a grande distância do centro da cidade (cerca de 10 quilômetros). Esses contatos, contudo, foram postti- vos porque aproximaram populaçãoEquipe de Saúde, passando a primeira a confiar e solicitar a aquipe para a orientação de seus problemas, por outro lado os alunos demonstraram interesse pelo trabalho em comunidade, quebrando o receio inicial.

No mês de abril de 1978 , a coordenação da Divisão de Enfermagem do Hospital "Dr. Celso Pierro", em fase de montagem, iniciou $o$ treinamento de pessoal para a futura Equipe de Saúde, o que veio de encontro a uma das necessidades da comunidade já que havia grande número de pessoas em idade economicamente ativa desempregadas. Através de membros das Sociedades de Amigos de Barros e do Grupo de Aposentados, foi feita intensa divulgação dos cursos oferecidos pela Pontifícia Universidade Católica de Campinas, utilizando distribuição de folhetos e afixação de cartazes em locais de afluência da população, a saber: açougues, mercearias, bares, pontos de ônibus, quitandas, praças, etc.

Do pessoal treinado, alguns foram contratados para os serviços iniciais do hospital e os demais encontram-se aguardando a inauguração para integrar-se ao quadro de funcionários.

Em junho de 1978 o Professor Titular do Departamento de Medicina Preventiva da Faculdade de Ciências Médicas, empenhou-se no sentido de montar um Ambulatório no centro dos bairros visados já que era a reivindicação maior da comunidade e havia necessidade premente desse tipo de campo de estágio para os alunos das Faculdades de Ciências da Saúde. Estruturou, paralelamente, o Instituto de Saúde Coletiva sob sua supervisão e composto por docentes das Faculdades de Ciências Médicas, Enfermagem, Serviço Social, Fisioterapia, Odontologia, Psicologia e elaborou o Programa Integrado de Assistência Progressiva à Saúde (PIAPS). Para a montagem do Ambulatório I - "Dr. Joaquim de Paula 
RESENDE, M.C. e Colaboradoras - Atuação da enfermagem em um sistema de saúde comunitária. Rev. Bras. Enf.; DF, 33 : 132-156, 1580.

Barreto Fonseca", foi alugada uma casa típica da Vila Castelo Branco e realizada reforma da mesma, adaptando-a para Ambulatório. O estudo dessa adaptação, bem como do material técnico mínimo necessário e rotina de atendimento, foram feitos em conjunto por docentes de Medicina e Enfermagem.

Em outubro de 1978 iniciou-se o atendimento ambulatorial de Clínica Médica, Obstetrícia e Ginecologia, Pediatria e Puericultura.

A divulgação na comunidade foi realizada por alunos, escolhendo aleatoriamente familias do bairro que recebiam um cartão, o qual deveriam apresentar por ocasião da primeira consulta.

A afluência ao Ambulatório foi mínima, crescendo à medida que conquistava a confiança da população.

No princípio de 1979 , com a montagem do Ambulatório II no Hospital "Dr. Celso Pierro", fol reformulado o atendimento comunitário para um programa realizado atualmente através do Ambulatório, com grupos de assistência Pré-Natal, de Saúde Escolar, de Puericultura, de Assistência à Hipertensos e de realização de visitas domiciliares para os casos em que isto se faz necessário.

Hoje podemos afirmar que a população solicita expontaneamente e, confia na equipe de saúde prestando também sua colaboração, e que os alunos das Faculdades de Ciências da Saúde aprenderam a utilizar seus conhecimentos em pról da comunidade menos favorecida, crescendo como profissionais, voltando seu interesse para a área de Saúde Pública e demonstrando entusiasmo pelo trabalho comunitário.

algumas Caractirifsticas da poptração

Os bairros Jardim Garcia, Vila Castelo Branco e Jardim Londres, possuem uma população calculada em torno de 10.000 individuos. Localizam-se na periferia da cidade de Campinas, contan- do com ruas asfaltadas, luz elétrica, água encanada, sistema de coleta de lixo e transporte (ônibus urbanos circulares), existem serviços de assistência médica.

Foi estudada uma amostra de 680 famílias compreendendo uma média de 3.400 familias.

Conclui-se que há uma média de cinco pessoas por familia, que o grau de instrução da maioria dos pais, situa-se no primário completo e incompleto $(68 \%)$; que as famílias procedem do Estado de São Paulo ( $76 \%)$, e de Minas Gerais $(10 \%)$; que os chefes de famílias em sua maioria são operários (14\% e motoristas $11 \%$ ) num total de oitenta e oito profissões encontradas; que a renda mensal familiar situa-se entre dois e cinco salários-mínimos (68\%); que $47,5 \%$ da população tem no máximo 14 anos e que a população em idade economicamente ativa reduz-se a $49,3 \%$. Foi a nda observado durante a pesquisa um número elevado de gestantes na árèa e grande número de indivíduos inativos pelas ruas.

Conclui-se que a população apresentava características que possibilitariam o desenvolvimento de um programa de assistência aliada ao processo ensino-aprendizado, preenchendo os objetivos iniciais. Com base nos dados da pesquisa, foi elaborado o Programa Integrado de Assistência Frogressiva à Saúde, prevendo níveis de atendimento no domícílio, na Unidade de Saúde e no Centro de Saúde Escolar.

\section{III - PROGRAMA INTEGRADO DE ASSISTENCIA PROGRESSIVA A SAÚDE (PIAPS)}

\section{JUSTITICATIVA}

A Pontifícia Universidade Católica de Campinas entende que é necessário oferecer a seus alunos oportunidade de ação comunitária, para que os mesmos possam aprender os aspectos ecológicos dos fenômenos saúde e doença. 
RESENDE, M.C. e Colaboradoras - Atuação d comunitá:ia. Rev. Bras. Enf.; DF, $33: 132-156,1530$.

Em assim fazendo, estará cooperando com a orientação governamental na área da saúde que advoga pela integração das Universidades com a comunidade, devolvendo a esta, sob a forma de prestação de serviços, um pouco do muito que dela recebe sob a forma de aprendizado.

Com o programa apresentado visamse os seguintes:

\section{ObJetTVOS}

1 - Treinar os alunos para que encarem a pessoa humana dentro de seu meio ambiente, estimulando seu interesse pelos problemas comunitários.

2 - Desenvolver nos alunos o espírito preventivista, indispensável ao tratamento integral dos pacientes e da comunidade.

3 - Desencastelar a Universidade, integrando-a à comunidade.

4 - Habituar os alunos ao trabalho de equipe com outros profissionais da saúde, pois a prevenção da doença exige a ação de equipes multiprofissionais.

5 - Desenvolver um modelo eficiente de prestação de serviços de saúde a baixo custo.

6 - Diminuir no prazo de 5 anos de $20 \%$ a mortalidade materna.

7 - Diminuir no mesmo prazo e percentagem a mortalidade infantil.

8 - Oferecer à comunidade um planejamento familiar condizente com os principios da moral cristã.

9 - Treinar os alunos no exercício da vigilância sanitária.

10 - Melhorar as condições de saúde da comunidade alvo, promovendo a saúde da família como meta básica.

\section{Procedimentos}

1 - Selecionar uma população alvo, que será ampliada à medida do possível, até atingir toda a população periférica da Cidade da Saúde.
2 - Divulgar na comunidade as disponibilidades de serviços, a fim de que possam: ser procurados pelos usuários.

3 - Organização de um arquivo de doentes e um banco de dados estatísticos.

4 - Participação dos alunos de todas as Unidades de Saúde da Universidade, conforme suas aptidões e preparos.

5 - Coletar informações que permitem a elaboração de estatísticas de saúde fidedignas, capazes de permitir a estimativa do estado de saúde da comunidade e a avaliação do PIAPS.

6 - Desenvolvimento de um setor de Relações Públicas, encarregado do entrosamento da comunidade com os sistemas de atendimento oferecidos.

7 - Prestar o atendimento médico em degraus, de maneira que os problemas mais simples sejam atendidos de maneira simples, evitando a sobrecarga dos niveis assistenciais mais sofisticados. Este modelo prevê o atendimento em quatro níveis, a saber:

Nivel I - Domicílio

O atendimento é feito por estudantes de Enfermagem; Serviço Social e de Medicina. Em caráter excepcional, alunos de outras Unidades poderão ser incluídos neste nível.

Serão Atribuições Deste Nível:

a) Controle de vacinação;

b) Educação materna sobre cuidados com a criança;

c) Educação para higiene e saneamento ambiental;

d) Registro de fatos vitais;

e) Descoberta de mulheres com suspeita de gestação;

f) Registro de morbidade;

g) Motivação para comparecimento aos niveis assistenciais superiores e encaminhamento aos mesmos, quando necessário; 
RESENDE, M. C. e Colaboradoras - Atuaçâo da enfermagem em um sistema de saúde comunitária. Rev. Bras. Enf.; DF, 33 : 132-156, 1580.

h) Controle do crescimento e estado nutricional da família;

i) Controle pós-partum dos partos atendidos por parteiras ou curiosas.

Cada estudante de Medicina coordenará $o$ atendimento de aproximadamente 10 (dez) famílias.

\section{Nível II - Unidade de Saúde}

O atendimento será prestado por estudantes de Medicina, Enfermagem, Odontologia e Educação Sanitária, sob a orientação de médicos, dentistas, enfermeiros e educadores sanitários lotados no Centro de Saúde Escola.

As Atribuições Deste Nível Serão:

a) Atenção à criança sadia;

b) Atenção à criança com diarréia ou desidratação grau I, estados gripais, piodermites leves, parasitoses;

c) Atenção às mulheres grávidas sem complicações;

d) Curativos simples e primeiros auxílios de pouca gravidade;

e) Recebimento e encaminhamento de material para exames;

f) Remoção de enfermos para o Centro de Saúde Escola;

g) Colaboração nos programas de paternidade responsável;

h) Elaboração de informações estatísticas e seu encaminhamento ao Centro de Saúde Escola;

i) Estudo da Saúde Dentária.

A Unidade de Saúde atenderá de 1.000 a 1.500 famílias.

Nível III - Centro de Saúde Escola

Nele estarão lotados pessoal médico, de enfermagem, odontológico, assistentes sociais, educadores sanitários, administrativo, de comunicação, auxiliar e de limpeza.

Os estudantes das diversas Unidades participarão das atividades do Centro de Saúde Escola na medida em que seus conhecimentos o permitirem.
Atividades do Centro de Saúde Escola:

a) Controle Pré-Natal de mulheres de mediano risco;

b) Atenção ambulatorial às complicações da gravidez, parto e pós-partum;

c) Exame médico de crianças sadias nos 30 primeiros dias de vida;

d) Controle periódico de crianças com baixo peso ou crescimento anormal;

e) Atenção ambulatorial de doentes de qualquer idade;

f) Colheita de material para exames especializados;

g) Encaminhamento ao Hospital Escola quando necessário;

h) Encaminhamento ao laboratório, dos materiais para exame.

i) Educação comunitária em termos de saúde;

j) Elaboração de programas de paternidade responsável e orientação na aplicação dos mesmos;

k) Atendimento odontológico preventivo e curativo;

1) Controle de doenças transmissíveis, com especial atenção para tuberculose, hanseníase e moléstias venéreas;

m) Atendimento preventivo e curativo, em âmbito ambulatorial, de doentes mentais;

n) Detecção de problemas nutricionais e elaboração de programas que objetivem soluções a cúrto prazo;

o) Organizar 0 arquivo central de doentes e o banco de informações estatísticas, em colaboração com o Centro de Processamento de Dados da Cidade da Saúde, capaz de possibilitar a elaboração contínua de relatórios estatísticos e epidemiológicos. Estes relatórios devem permitir a avaliação dinâmica do estado de saúde da população alvo, assim como da qualidade dos serviços prestados e dos resultados obtidos em termos de aprendizado;

p) Desenvolver atividades visando à assistência médico-sanitária e à educação sanitária da população alvo; 
RESENDE, M.C. e Colaboradoras - Atuação da enfermagem em um sistema de saúde comunitária. Rev. Bras. Enf.; DF, 33 : 132-156, 1980.

q) Vacinações;

r) Auxiliar e orientar as atividades dos niveis inferiores.

Nivel IV - Hospital Escola

Atenderá os casos que exijam maior sofisticação diagnóstica e terapêutica e realizará os exames subsidírios solicltados pelos níveis inferiores.

O Programa Integrado de Assistência Progressiva à Saúde será supervislonado, em todos os nivels, por elementos do Corpo Docente das diversas Unidades envolvidas no mesmo.

\section{RECURSOS}

Estarão engajadas no PIAPS todas as Unidades da área de saúde da Pontifícia Universidade Católica de Campinas em especial as de Ciências Médicas, Enfermagem, Odontologia, Serviço Social e Psicologia.

Todas as atividades serão coordenadas e supervisionadas pelo Instituto de Saúde Coletiva.

Os recursos financeiros serão procurados mediante convênios com Institulçōes Assistencials Oficiais e auxilios de entidades não brasileiras.

\section{IV - PROGRAMAS EM DESENVOLVIMENTO}

Como parte do Programa Integrado de Assistência Progressiva à Saúde (PIAPS), estão sendo desenvolvidos os seguintes programas:

\section{AMBULATORIO}

Atende à população com programa de grupos que será abordado em sequêncla. Como referimos anteriormente, houve adaptação de uma casa residencial e sentimos a necessidade de anexar ao trabalho a planta física do Ambulatório (Anexo 1).

O registro das atividades é realizado em livro de atas das reuniōes de cada grupo; flchas próprias para os dife- rentes programas (Saúde Escolar (Anexo 2), Puericultura (Anexo 3), Gestantes (Anexo 4), Hipertensos (Anexo 5), fichas de identificação dos membros de todos os grupos (Anexos 6 e 7), impressos para registro das atividades da equipe e livro de registro de consultas.

0 atendimento se dá no horário das 8 às 18 horas, sendo que o das 8 às 12 horas, é realizado somente pela Assistência Social e o das 12 às 18 horas, por todos os membros da equipe de saúde.

Quando elementos da comunidade solicitam atendimento que não pode ser proporcionado nos grupos (ex.: problema de clínica médica), o caso é encaminhado para o Ambulatório II do Hospital "Dr. Celso Pierro".

O Ambulatório oferece ainda através do Serviço de Enfermagem: Primeiros Socorros (para a comunidade e os alunos da Escola Estadual de $1 .^{\circ}$ grau do bairro Castelo Branco) curativos, medicação de urgência, aplícação de medicação intra-muscular e endovenosa (com receita médica) e de anatox-tetânico. Fol solicitada aos órgãos competentes e já aprovada, a distribuiçẳo, em futuro próximo, de gestal e leite em pó, bem como a vacinação em geral da população.

Nos dias de intervalo entre o atendimento dos grupos, seus membros podem solicitar assistência do Ambulatório sempre que assim se fizer necessárío.

Cabe alnda ao ambulatório, o controle e notificação, às autoridades sanitárias, das doenças transmissíveis que incidem na área.

\section{EQUIPE DE SAODE}

Atualmente a Equipe de Salide conta com médicos, enfermeiras, assistente social, psicóloga, servente, alunos, voluntários, e, quando necessário é solicitado a presença da nutricionista. Semanalmente são reallzadas reuniōes para balanço das atividades realizadas 
RESENDE, M.C. e Colaboradoras - Atuação da enfermagem em um sistema de saúde comunitária. Rev. Bras. Enf.; DF, 33 : 132-156, 1880.

pela equipe multiprofissional e dos problemas que surgiram durante a semana. Há uma troca de experiências e uma orientação conjunta do trabalho, sob liderança do médico-chefe do Ambulatório I.

Detalharemos a seguir, as funções especificas de cada membro da equipe, que se interligam e completam, visando unicamente o melhor atendimento da população e um padrão elevado de ensino para os alunos da área de Ciênclas da Saúde.

\section{MEDICINA}

- Executar o exame clínico dos cllentes e realizar o diagnósico.

- Solicitar exames complementares e prescrever o tratamento.

- Encaminhar clientes para outros profissionais da equipe, conforme necessidade.

- Participar da prevenção de moléstias e orientação sanitária.

- Supervisão dos programas desenvolvidos no Ambulatório e comunidade.

- Orientação e supervisão dos alunos da Faculdade de Ciências Médicas da Pontifícia Universidade Católica de Campinas.

- Realização de pesquisas na área.

\section{ENFERMAGEM}

- Proteção e promoção da saúde individual e da coletividade.

- Encaminhamento de clientes para outros profissionais da equipe.

- Participação nos programas de saúde do Ambulatório.

- Educação sanitária individual, em grupo e da comunidade.

- Supervisão e orientação de alunos da Faculdade de Enfermagem.

- Controle de Enfermagem dos cllentes atendidos no Ambulatório.

- Orientação de pacientes, na comunidade, com alta hospitalar.

- Treinamento e supervisão da equipe de Enfermagem.
- Administração do Serviço de Enfermagem do Ambulatório.

- Prestação de cuidados de Enfermagem no Ambulatório e no domicílio.

- Realização de levantamentos e pesquisas de interesse para as atividades da Equipe de Saúde.

\section{SERVIÇO SOCIAL}

- Orientação individual e familiar quanto à: documentação, previdência social, trábalho, conduta social, desestruturaçã̀o pessoal.

- Encaminhamento de clientes para os demais membros da Equipe de Saúde, para o Ambulatório II ou para outros recursos da comunidade.

- Visita domiciliar para avaliar as condições da familia e solicitar a part1cipação de outros profissionais da Equipe.

- Supervisão e orientação de estagiárias da Faculdade de Serviço Social da Pontifícia Universidade Católica de Campinas.

- Treinamento de agentes de saúde.

- Contato com institulções de interesse na execução de seu trabalho.

- Participação nos programas desenvolvidos no Ambulatório.

\section{PSICOLOGA}

- Orientação a indivíduos, grupos e familias nos aspectos referentes à psicologia. fissional.

- Participar da equipe muitipro-

\section{NUTRICIONISTA}

- Oferecer orlentação a indịvíduos, grupos e famílias no campo da nutrlção, quando sollcitada por profissional da equipe.

\section{SERVFNTE}

A servente é um elemento da comunidade que está sendo trelnado também para exercer algumas funços de atendente de enfermagem, a sabier: 
RESENDE, M.C. e Colaboradoras - Atuação da enfermagem em um sistema de saúde comunitária. Rev. Bras. Enf.; DF, 33 : 132-156, 1680.

- Responsável pela ordem e limpeza do Ambulatório;

- Atende telefone, transmite recados e faz o serviço de copa;

- Preparo das salas de consulta;

- Lavar e acondicionar o material utilizado nas técnicas de enfermagem;

- Participa do Programa de Saúde Escolar, conduzindo os alunos, com suas fichas desde a Escola Estadual até - Ambulatório e vice-versa, e exercendo vigilância sobre os mesmos na sala de espera durante os exames;

- Age na comunidade detectando problemas sociais ou de saúde e encaminhando os clientes para o Ambulatório.

\section{VOLUNTARIOS}

São elementos que residem na comunidade e que são treinados para, como agentes de saúde, prestar cuidados primários e encaminhar pessoas da comunidade para 0 atendimento ambulatorial. São um elo de união entre a equipe de saúde e a comunidade. Não possuem vínculo empregatício com a Universidade.

\section{INTEGRACGA DOCENTE ASSISTENCIAL}

A integração docente assistencial tem sido realizada através de um método simples e prático. A enfermeira de campo ministra aulas de Saúde Pública na Faculdade de Enfermagem e supervisiona o estágio de alunos no Ambulatório e na comunidade, por sua vez os docentes de Enfermagem participam da experiência do grupo de Saúde Pública integrando-se no trabalho desenvolvido, e a enfermeira responsável pelo Departamento de Saúde Pública da Faculdade de Enfermagem supervisiona o Ambulatórío I e atua com os alunos, em contato direto com a comunidade. Os médicos da equipe são docentes da Faculdade de Clênclas Médicas e prestam assistência médica, com os alunos, no smbulatório.
As estagiárias da Faculdade de Serviço Social, são supervisionadas pela assistente social de campo.

\section{PROGRAMAS}

Pré-Natal - Está sendo desenvolvido com 18 (dezoito) gestantes divididas por $10^{\circ}, 2^{\circ}$ e $3 .^{\circ}$ trimestre de gravidez. A cada 15 (quinze) dias as mesmas apresentam-se ao Serviço de Enfermagem que: realiza o controle PréNatal, orienta sobre a higiene da gravidez e encaminha para consulta médica os casos em que isto se faz necessário. A seguir é realizada reunião com participação da Medicina e Enfermagem que apenas coordena a reunião, estimulam a discussão de problemas (relacionados com a gravidez, parto e puerpério) pelas próprias gestantes, interferindo somente para esclarecer ponto em dúvida.

\section{SAÚDE ESCOLAR}

Em conjunto com a díreção da Escola Estadual de 1.0 Grau "Professor Antônio Fernandes Gonçalves", do bairro Castelo Branco, e com a colaboração de sua orientadora pedagógica, a Equipe de Saúde vem realizando a triagem de 1.600 (um mil e seiscentos) crianças na faixa etária dos 7 (sete) aos 16 (dezesseis) anos, em atendimento ambulatorial, duas vezes por semana.

A triagem teve início com classesproblema e de repetentes, selecionados pela orientadora pedagógica. As professoras, orientadas pela psicóloga, preparam o histórico escolar, relatam - relacionamento-comportamento-aproveitamento dos alunos em ficha própria (Anexo 2) e aplicam o teste de audiometria. Em grupos os alunos, são conduzidos ao Ambulatório I, pela servente do mesmo, juntamente com o histórico escolar, a flcha e o resultado do teste de audiometria.

Passam pelo Serviço de Enfermagem, onde é verfficado: peso, estatura, 
RESENDE, M. C. e Colaboradoras - Atuação da enfermagem em um sistema de saúde comunitária. Rev. Bras. Enf.; DF, 33 : 132-156, 1580.

perimetro cefálico, temperatura, pressão arterial, e é aplicado teste de optometria. São encaminhados ao médico que, em conjunto com seus alunos, realiza o exame clínico e orienta os pais, quando necessário, em conjunto com a psicóloga e demais membros da Equipe de Saúde solicitados de forma especial.

A Assistente Social realiza entrevista com as crianças e suas respectivas māes, nos casos selecionados para visita domiclliar.

\section{PROGRAMA DE PUERICULTURA}

Cobre a faixa etária de $0-24$ meses. Conta com giupos divididos por faixa etária, a saber:

$$
\begin{aligned}
& 0-2 \text { meses } \\
& 3-4 \text { meses } \\
& 5-7 \text { meses } \\
& 6 \text { meses } \\
& 12-12 \text { meses num total de } 156 \\
& \text { crianças } \\
& 15-24 \text { meses. } \\
& \text { o controle para cada grupo é men- }
\end{aligned}
$$
sal. A Enfermagem verifica o peso, estatura e perf́metro cefálico. Elabora o gráfico ponđo-estatural da criança para comparação com o mês anterior e discussão na reunião. Verifica se a imunização da criança está correta e encaminha para vacinação quando necessário. Encaminha para controle médico os casos em que isto se faz necessário. A seguir é reallzada a reunião sendo que os problemas são colocados e discutidos pelas próprias mães, que assim trocam experiências. As mães recebem tarefas que devem ser relatadas na próxima reunião. A Enfermagem e a Medicina estão presentes apenas para induzir a discussão dos problemas relacionados com os cuidados com a criança, e auxiliar na solução de problemas.

\section{GRUPO DE HIPERTENSOS}

A Enfermagem realiza semanalmente palestras de orientação sobre hipertensão e outros problemas de saúde co- locados por um grupo de 26 hipertensos, também é responsájvel pelo controle da pressão arterial edema-pesoqueixas-dietas-medicação-repouso (vide Anexo 5), seleciona dias em que os diferentes membros do grupo devem passar por consulta médica.

Quinzenalmente o médico comparece à reunião para esclarecer sobre os problemas apresentados.

\section{VISITA DOMICILIAR}

E realizada nos casos em que se faz necessária a orientação da família ou a prestação de serviços no domicílio para os clientes atendidos nos programas desenvolvidos no Ambulatório I.

O Serviço Social faz o primeiro contato avaliando as condições sócio-econômicas da família e encaminhando a continuidade da assistência segundo as necessidades apresentadas, para os outros profissionais da equipe, a saber: Enfermeira, Nutricionista ou Psicóloga.

\section{CONCLUSAO}

Tem sido muito positivo para a Faculdade de Enfermagem, incluindo docentes e alunos, o contato com a população e com profissionais de outras áreas. Após praticamente um ano de atividades pode ser observado um enriquecimento profissional da equipe e uma prálica que nos leva a crescer e estimular o crescimento da comunidade atingindo os objetivos inicialmente propostos.

A avaliação dos resultados de nosso trabalho será feita em futuro próximo, mas os efeitos observados até o momento, na equipe e na comunidade, nos dão a certeza de estarmos direcionando corretamente o processo ensino-aprendizagem de Saúde Pública e a assistência à comunidade, 0 que nos estimula a intensificar, aprofundar e expandir as atividades na Equipe de Enfermagem de Saúde Pública. 
RESENDE, M.C. e Colaboradoras - Atuação da enfermagem em um sistema de saúde comunitária. Rev. Bras. Enf.; DF, 33 : 132-156, 1880.

ANEXO 1

AMbulatorio I Dr. JOAqUim de PAULA BARRETO FONSECA

Rua Humberto de Campos n. ${ }^{\circ} 184$ - Vila Castelo Branco - Campinas

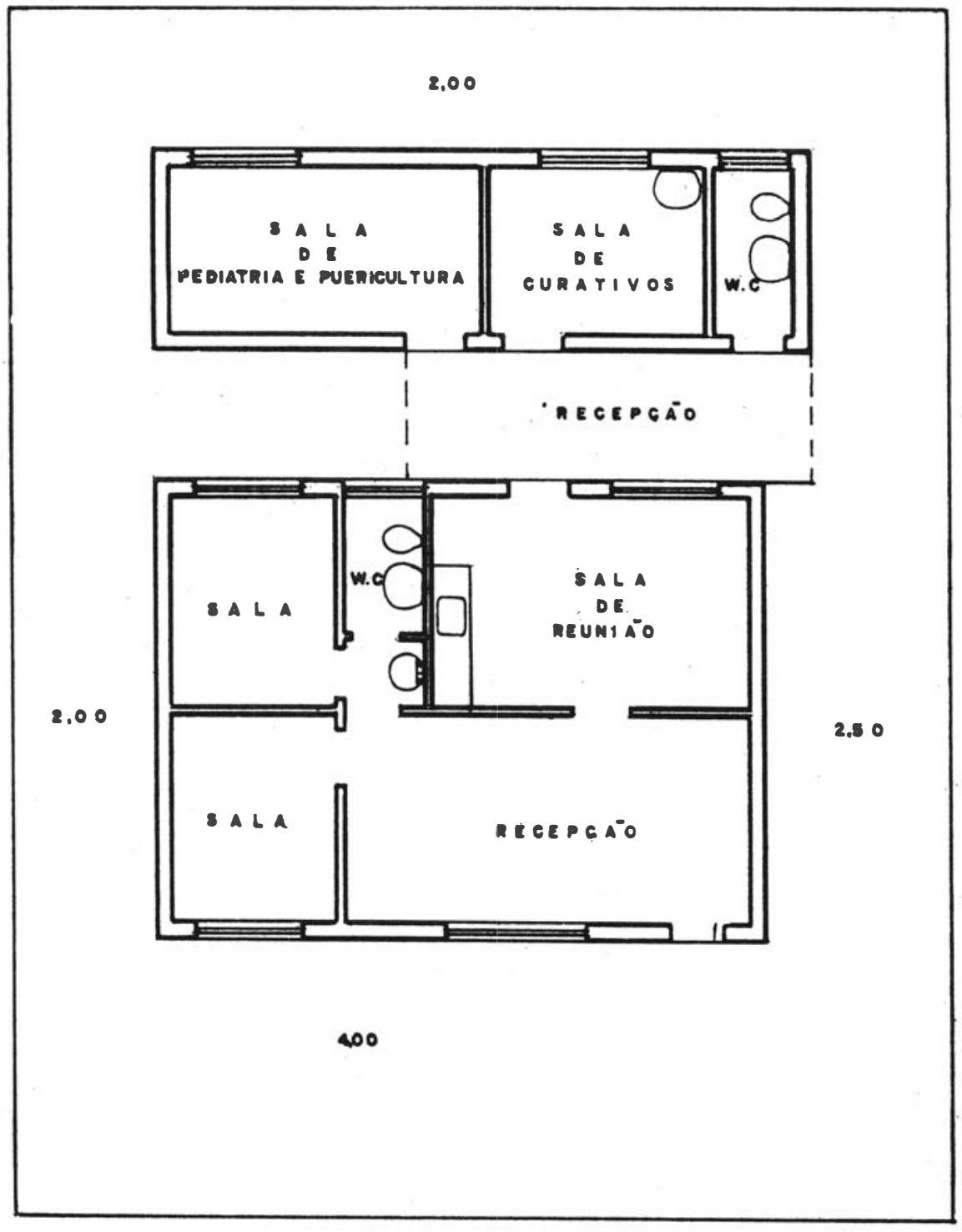

PLANTA FISICA

EsC. $1: 100$ 
RESENDE, M.C. e Colaboradoras - Atuação da enfermagem em um sistema de saúde comunitária. Rev. Bras. Enf.; DF, 33 : 132-156, 1980.

ANEXO 2

\section{P R O G RA MA D E SA U D E E S C O LA R \\ CONVOCACG O DAS MAES}

A Sra. $(\ldots \ldots \ldots \ldots \ldots \ldots \ldots \ldots \ldots \ldots \ldots \ldots \ldots \ldots \ldots \ldots \ldots \ldots \ldots$ deverá comparecer ao . no dia ................ às horas, para acompanhar o atendimento de seu filho.

de de

\section{Assinatura da Professora}

ANEXO 2

$$
\text { C O N V O C A A } \mathrm{O}
$$

Comunicamos, que seu filho passará por exame médico no dia $\ldots . . / \ldots / \ldots \ldots$ pedimos não deixá-lo faltar neste dia, e enviar a caderneta de vacinação.

Se quiser venha com seu filho.

de de

Assinatura da Professora

ANEXO 2

\section{SA U D E E S C O L A R}

FICHA N.O

ESCOLA:

BAIRRO

NOME DO ALUNO:

IDADE:

SEXO

ENDEREÇO:

COR:

NOME DO PAI:

NOME DA MAE: 
RESENDE, M. C. e Colaboradoras - Atuaçāo da enfermagem em um sistema de saúde comunitária. Rev. Bras. Enf.; DF, 33 : 132-156, 1980.

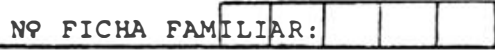

FICHA DE ADULTOS: MASCULINO $\square$ FEMININo. $\square$

OAREULEA

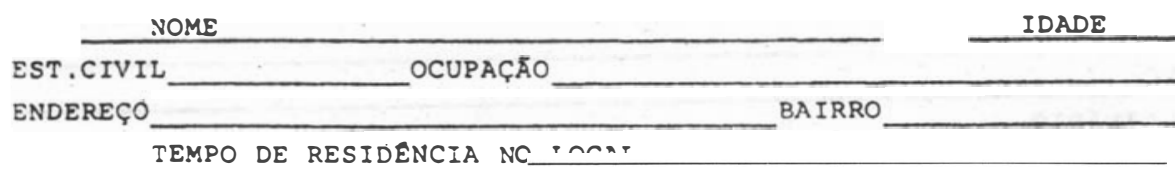

ANTECEDENTES MORBIDOS PESSOAIS

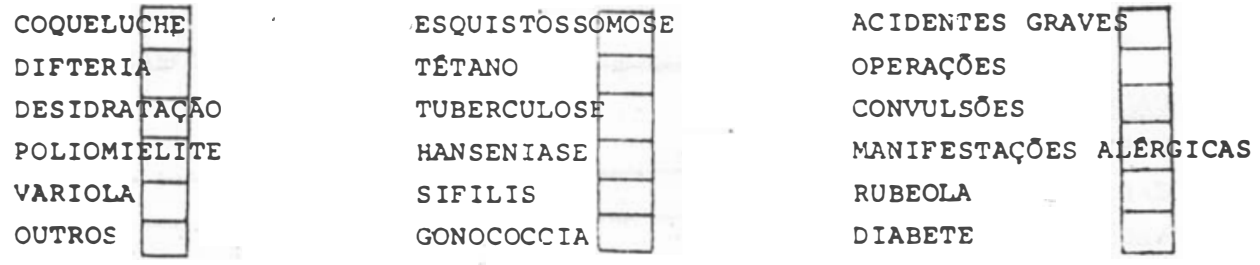

ANTECEDENTES MOBIDOS FAMILIARES

\begin{tabular}{|c|c|c|c|}
\hline TUBERCULOSE & REAÇŌES ALERG & CAS & MAL FORMACOES \\
\hline HANSEN IASE & SÍFILIS & & INTERN . PSIQUIATRICAS \\
\hline CONVULSŌES & ALCOOLISMO & & DIABETES \\
\hline
\end{tabular}

OUTROS

ANTECEDENTES GINECOLOGICOS

MENARCA (19 menstruaçāo) $\square$ ANOS CICLOS REGULARES $\square$

NP DE GESTAÇÕES ANTERIORES $\square$ NP DE ABORTOS $\square$

NO PARTOS A TERMO $\square$ PREMATUROS $\square$ NATIVIVOS $\square$ NATEMORTOS

GÊMEOS $\square$. NORMAIS $\square$ CESAREA $\square$ FORCEPS $\square$

ULTIMO PARTO HA $\square$ MESES $\square$ DIA DA ULTIMA MENSTRUAÇÃO $\square \square$

\section{ANTICONCEPCุÃO}

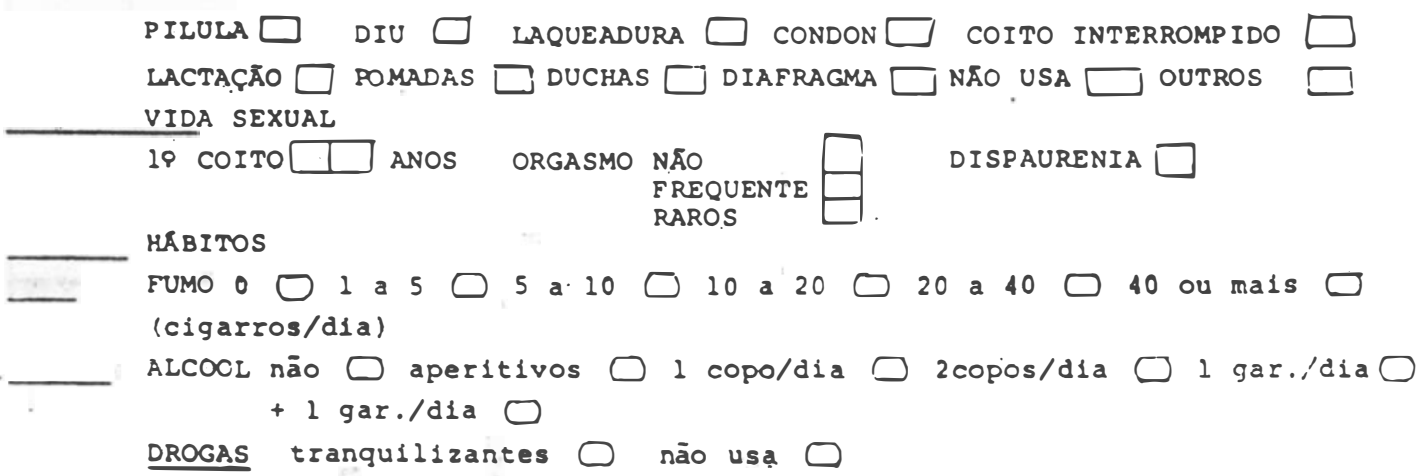


RESENDE, M.C. e .Colaboradoras - Atuação da enfermagem em um sistems de safod comunitária. Rev. Bras. Enf.; DF, 33 : 132-156, 1980.

\section{CONCLUSAO}

\section{HISTORICO ESCOLAR}

COMPORTAMENTO:

APROVEITAMENTO:

RELACIONAMENTO C/COLEGAS:

OBS.:

CONCLUSAO:

IMPRESSAO FINAL E ORIENTACAO 
RESENDE, M. C. e Colaboradoras - Atuação da enfermagem em um sistema de saúde comunitária. Rev. Bras. Ene.; DF, 33 : 132-156, 1980.

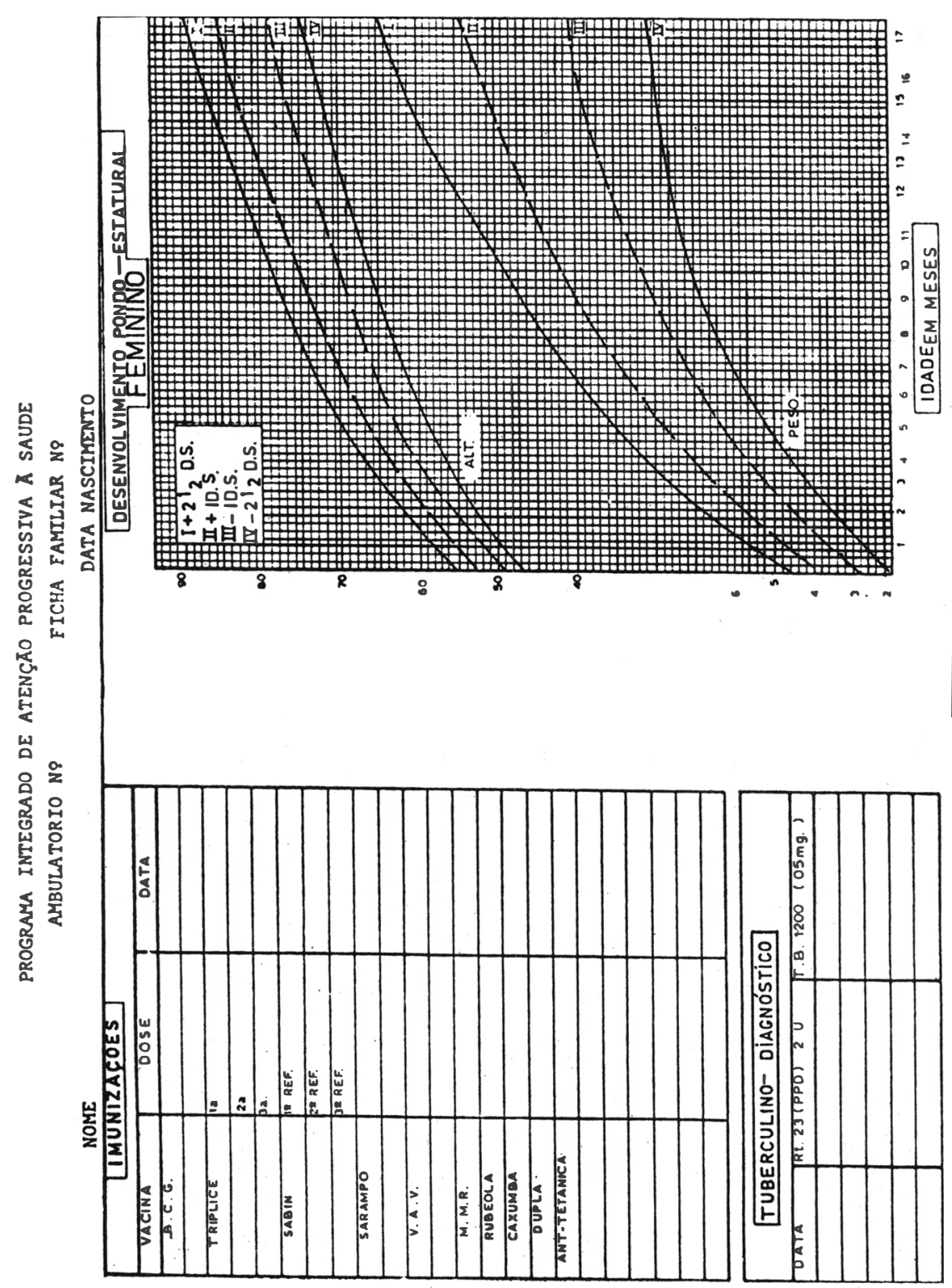


RESENDE, M.C. e Colaboradoras - Atuação da enfermagem em um sistema de saúde comunitária. Rev. Bras. Ene.; DF, 33 : 132-156, 1880.
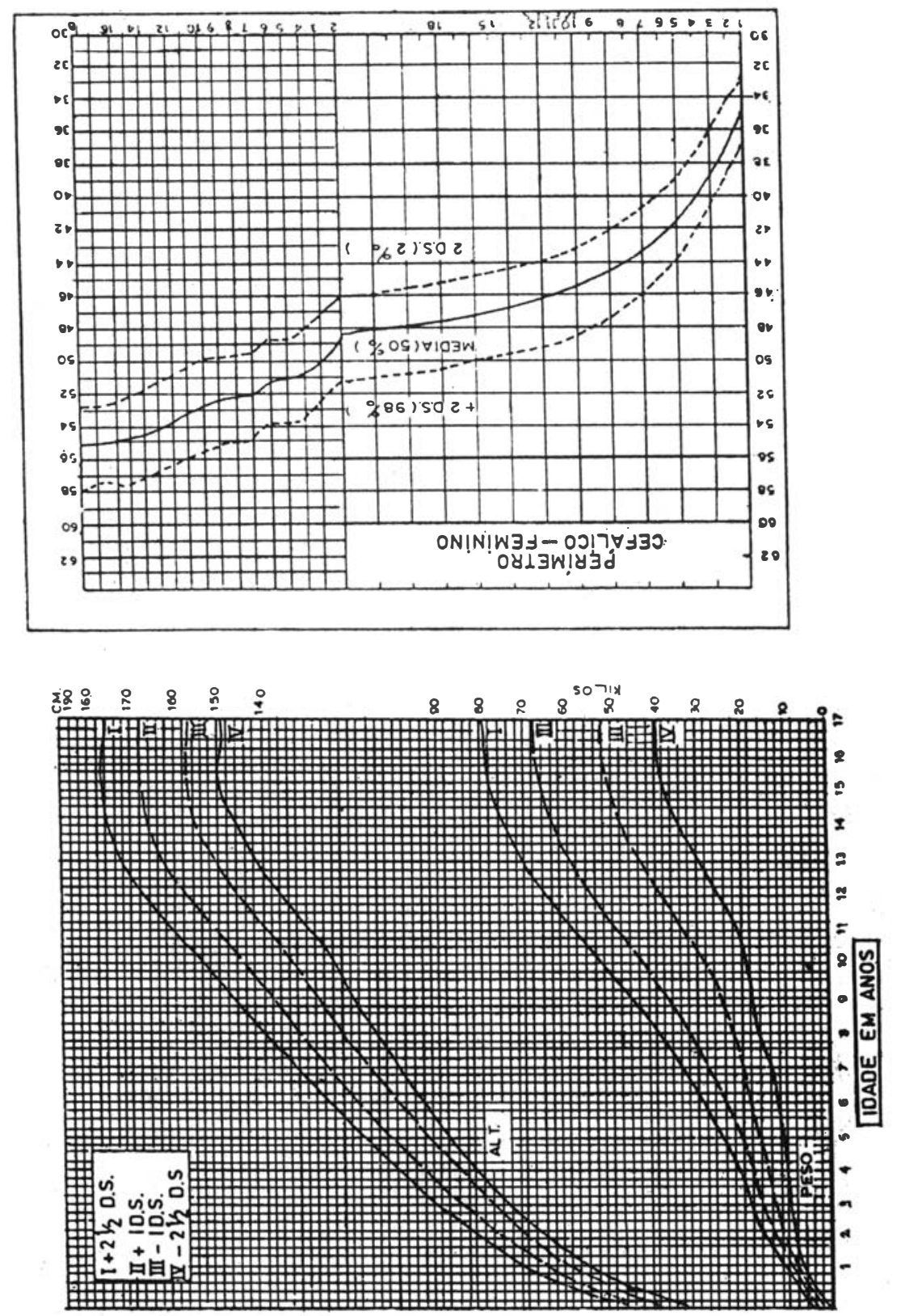
RESENDE, M. C. e Colaboradoras - Atuação da enfermagem em um sistema de saúde comunitária. Rev. Bras. Ent.; DF, 33 : 132-156, 1980.

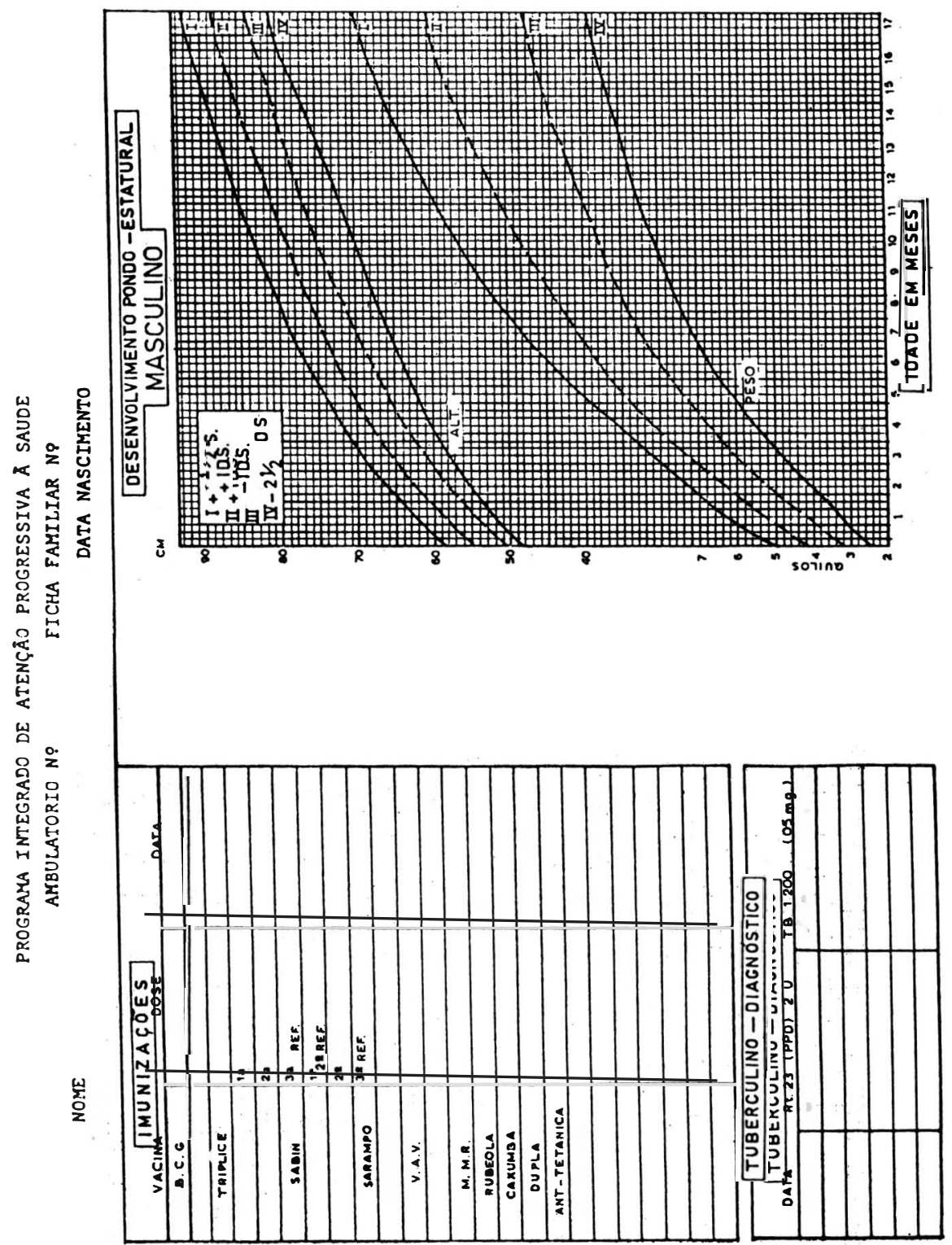


RESENDE, M.C. e Colaboradoras - Atuação da enfermagem em um sistema de saúde comunitária. Rev. Bras. Enf.; DF, 33 : 132-156, 1930.
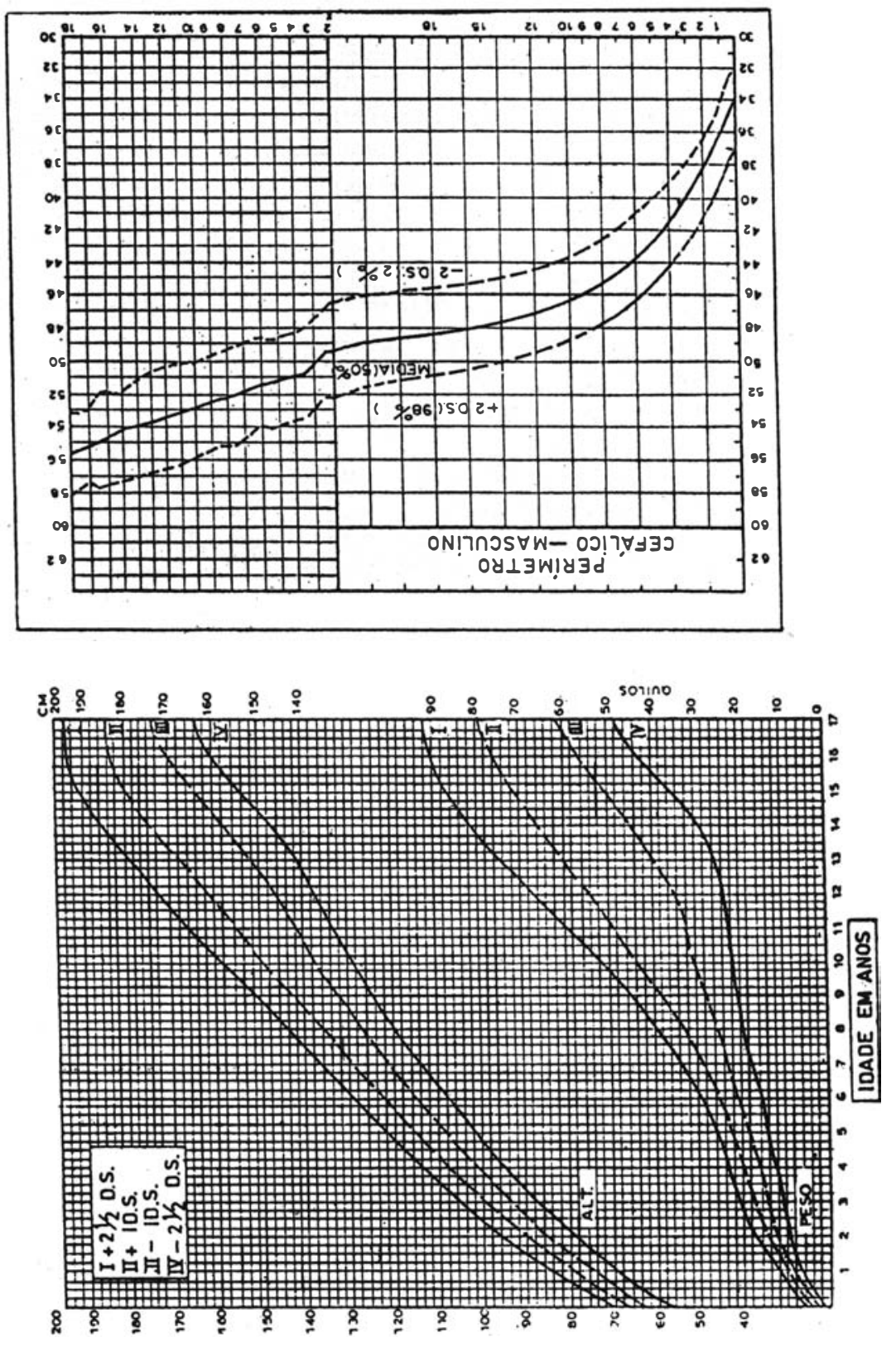
RESENDE, M. C. e Colaboradoras - Atuaçāo da enfermagem em um sistema de saúde comunitária. Rev. Bras. Ene.; DF, 33 : 132-156, 1980.

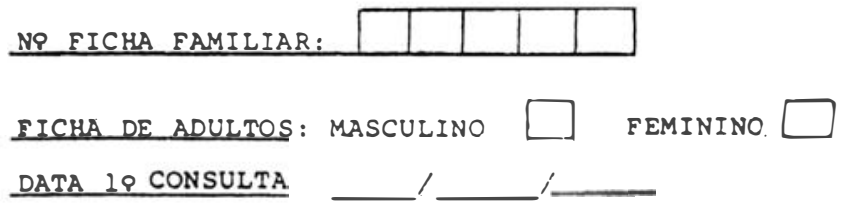

NOME IDADE

EST. CIVIL OCUPACุÃO

ENDEREÇO BA IRRO

TEMPO DE RESIDENCIA NO LOCAL

\section{ANTECEDENTES MORBIDOS PESSOAIS}

COQUELUCHE DIFTERIA

DESIDRATAÇÃO POLIOMIELITE

VARIOLA

OUTROS

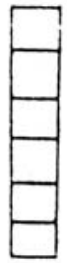

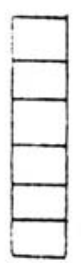

ACIDENTES GRAVES OPERACৃŌES CONVULSŐES MANIFESTAÇŌES ALERGICAS RUBEOLA DIABETE

ANTECEDENTES MOBIDOS FAMILIARES

TUBERCULOSE

HANSEN IASE

CONVULSŌES

OUTROS

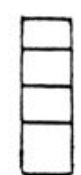

REAÇŌES ALERGICAS SIFILIS

ALCOOLISMO

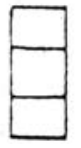

MAL FORMACOẼS INTERN . PSIQUIATRICAS DIABETES

\section{ANTECEDENTES GINECOLOGICOS}

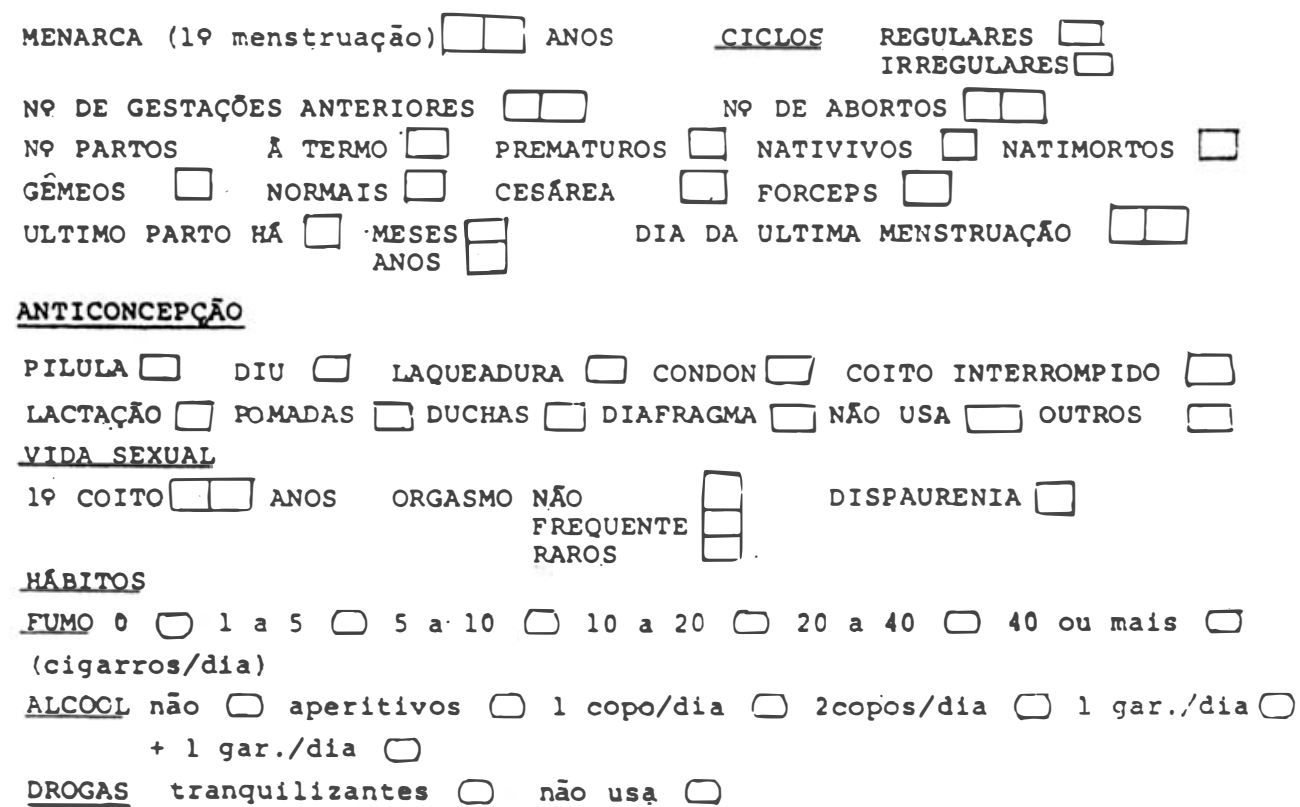


RESENDE, M. C. e Colaboradoras - Atuação da enfermagem em um sistema de saúde comunitária. Rev. Bras. Enf.; DF, 33 : 132-156, 1980.

QUEIXA PRINCIPAL

INTER. DIF. APARELHOS

CABEÇA

CARDIO RESP.

DIGESTIVO

GENITO. URINARIO

NEURO-MOTOR

EXAME FISICO

P.A P.

T MUCOSAS

DADOS POSITIVOS

CABECA

CARDIO RESPIRATORIO

ABDŌMEM

GENITO. URINARIO

TOOUE RETAL

VAGINAL

CONDUTAS:

DIAGNOSTICO FINAL $\square \square \square \square$

D CONHECIDOS OU FAMILIARES COM A MESMA DOENÇA

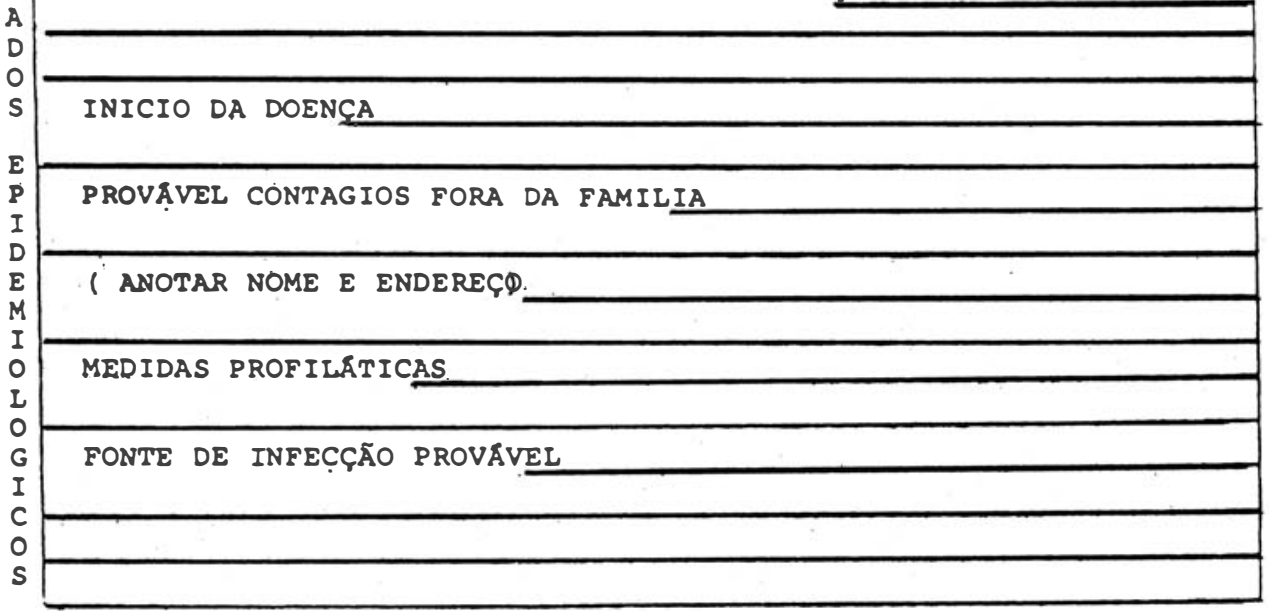

RESULTADO DOS EXAMES: 
RESENDE, M.C. e Colaboradoras - Atuação da enfermagem em um sistema de saúde comunitária. Rev. Bras. Enf.; DF, 33 : 132-156, $15 \cdot 80$.

PRE NATAL E PUERPERIO

NOME

DATA DA ULTIMA MENSTRUAÇÃO DIA

NO F.FAMILIAR MES ANO

\begin{tabular}{|l|l|l|l|l|l|l|l|l|}
\hline DATA & QUEIXAS & SEM.GES & PESO: & P.A. & A.U. & CIR. & MOV.FET FOCO & EDEMA \\
\hline
\end{tabular}

\begin{tabular}{l|llllllllllll|}
\hline & & & & & & & & & \\
\hline & & & & & & & & & \\
\hline & & & & & & & & & \\
\hline & & & & & & & & & \\
\hline & & & & & & & & & \\
\hline & & & & & & & & & \\
\hline & & & & & & & & & \\
\hline & & & & & & & & & \\
\hline & & & & & & & & & \\
\hline & & & & & & & & & & \\
\hline & & & & & & & & & & \\
\hline & & & & & & & & & & \\
\hline & & & & & & & & & & \\
\hline & & & & & & & & & & \\
\hline & & & & & & & & & & & \\
\hline
\end{tabular}

O.D. (P.CONSULTA)

TOQUES

MED ICAMENTOS

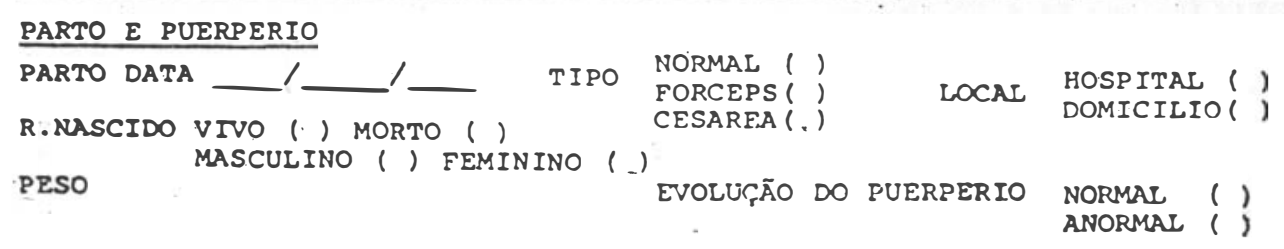


RESENDE, M. C. e Colaboradoras - Atuação da enfermagem em um sistema de saúde comunitária. Rev. Bras. Enf.; DF, 33 : 132-166, 1930.

ANEXO 5

\section{PROGRAMA DE hipertensos}

N.O FF.

NOME:

IDADE:

ENDERECO:

BAIRRO:

DATA: $\ldots \ldots / \ldots / \ldots / \ldots /$ GRUPO:

HA QUANTO TEMPO SABE QUE TEM HIPERTENSÃO?

QUAIS MEDICAMENTOS USA ATUALMENTE? (dose e nome do remédio)

FALTA DE AR ( )

BATEDEIRA ( )

DOR DE CABEÇA ( )

TONTURAS ( )

ZUMBIDOS ( )

OBSERVAÇAO:

\section{QUEIXAS}

PROBLEMAS VISUAIS ( )

DESMAIOS ( )

INCHACO NAS PERNAS ( )

SANGRAMENTO NASAL ( )

FORMIGAMENTOS

RESULTAdO DOS EXAMES: 
RESENDE, M.C. c Colaboradoras - Atuaçāo da enfermagem em um sistema de saúde comunitária. Rev. Bras. Enf.; DF, 33 : 132-156, 1980.

CONTROLE SEMANAL

NO FF.

PF EDEl:A PESO QUEIXAS/OBS.

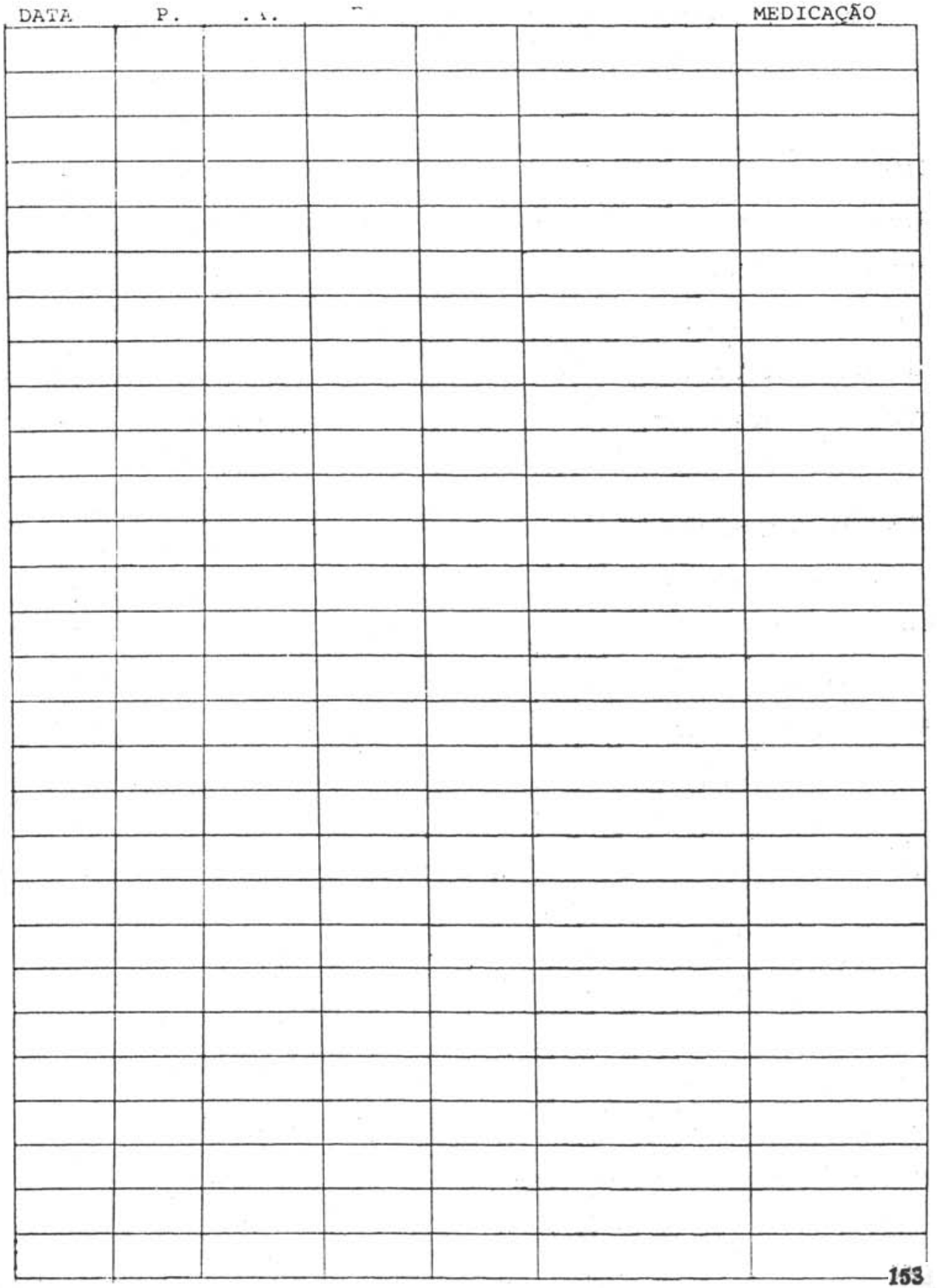


RESENDE, M. C. e Colaboradoras - Atuação da enfermagem em um sistema de saúde comunitária. Rev. Bras. Enf.; DF, 33 : 132-156, $15 \cdot 80$.

ANEXO 6

PONTIFtCIA UNIVERSIDAdE CATOLICA DE CAMPINAS

PROGRAMA INTEGRADO DE ATENÇAOO PROGRESSIVA A SAÚDE

FAMfLIA N.o

NOME:

SEXO:

COR:

DATA DE NASCIMENTC:

NATURALIDADE:

RESIDENCIA ATUAL:

BAIRRO:

INST. DE ASSIST. M色ICA:

ESTUDANTE RESPONSAVEL: .RA:

.RA:

MUDANÇAS DE ENDEREÇO:

OBSERVAÇAO:

PROGRAMA: 
RESENDE, M.C. e Colaboradoras - Atuação da enfermagem em um sistema de saúde comunitária. Rev. Bras. Enf.; DF, 33 : 132-156, 1980.

ANEXO 7

PONTIFICIA UNIVERSIDADE CATÓLICA DE CAMPINAS

PROGRAMA INTEGRADO DE ATENÇÃO PROGRESSIVA A SAÚDE

PROGRAMA:

FAMILIA:

NOME:

SEXO:

IDADE:

COR:

ESTADO CIVIL:

\begin{tabular}{|c|c|c|c|c|}
\hline DATA & ATIVIDADE & COMPARECEU & FALTOU & RETORNO \\
\hline & . & & & \\
\hline & & & & \\
\hline & & & & \\
\hline & & & & \\
\hline & & & & \\
\hline & & & & \\
\hline & & & & \\
\hline & & & & \\
\hline & & & & \\
\hline & & & & \\
\hline & & & & \\
\hline & & & & \\
\hline & & & & \\
\hline & & . & & \\
\hline r & & . & & \\
\hline & & & & \\
\hline & & & & \\
\hline & & & & \\
\hline & & & & \\
\hline & & & & \\
\hline
\end{tabular}


RESENDE, M.C. e Colaboradoras - Atuaçāo da enfermagem em um sistema de saúde comunitária. Rev. Bras. Enf.; DF, 33 : 132-156, ,1530.

\section{REFERENCIAS BIBLIOGRAFICAS}

NOGUEIRA, MJC - O Hospital, sua função na comunidade $e$ o papel da Enfermagem de Saúde Pública - Rev. Enfermagem em Novas Dimensões - Págs. 37, 41 - Vol. 1 - N. ${ }^{\circ} 1$ - Março-Abril de 1975 - SP.

OMS - Organização Mundial de Saúde Pública - Profilaxia das Doenças Transmissiveis - 11." Ed. - Washington D.C. - 1973.

KING, Dr. Maurice - Medical Care in Reveloping Countries - Nairobi - 1966.
LEPARGNEUR, Hubert - A Igreja $e$ os Problemas Atuais de Saude - Rev. O Mundo da Saúde - 3, 25, 29 - Sáo Paulo - 1977.

ADAMI, Dra. Nilce Piva - A Funçāo da Supervisora em Enfermagem de Saude Pública - Rev. Brasileira de Enfermagem - Ano XXVIII - N.0 1 - Janeiro-Março - 1976.

PERETTA, José Maria Marlet - Saude da Comunidade, São Paulo - Ed. Mac Graw-Hill do Brasil - 1976. 